\title{
Confidence intervals for the difference between the coefficients of variation of Weibull distributions for analyzing wind speed dispersion
}

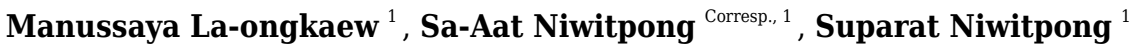 \\ ${ }^{1}$ Department of Applied Statistics, King Mongkut's University of Technology North Bangkok, Bangkok, Thailand \\ Corresponding Author: Sa-Aat Niwitpong \\ Email address: sa-aat.n@sci.kmutnb.ac.th
}

Wind energy is an important renewable energy source for generating electricity that has the potential to replace fossil fuels. Herein, we propose confidence intervals for the difference between the coefficients of variation of Weibull distributions constructed using the concepts of the generalized confidence interval (GCl), Bayesian methods, the method of variance estimates recovery (MOVER) based on Hendricks and Robey's confidence interval, a percentile bootstrap method, and a bootstrap method with standard errors. To analyze their performances, their coverage probabilities and expected lengths were evaluated via Monte Carlo simulation. The simulation results indicate that the coverage probabilities of $\mathrm{GCl}$ were greater than or sometimes close to the nominal confidence level. However, when the Weibull shape parameter was small, the Bayesian- highest posterior density interval was preferable. All of the proposed confidence intervals were applied to wind speed data measured at 90-meter wind energy potential stations at various regions in Thailand. 
Confidence intervals for the difference between the coefficients of variation of . Weibull distributions for analyzing wind - speed dispersion

${ }_{5}$ Manussaya La-ongkaew, Sa-Aat Niwitpong, and Suparat Niwitpong

6 Department of Applied Statistics, Faculty of Applied Science, King Mongkut's

7 University of Technology North Bangkok, Bangkok, Thailand

Corresponding author:

Sa-Aat Niwitpong

Email address: sa-aat.n@sci.kmutnb.ac.th

\begin{abstract}
Wind energy is an important renewable energy sources for generating electricity that has the potential to replace fossil fuels. Herein, we propose confidence intervals for the difference between the coefficients of variation of Weibull distributions constructed using the concepts of the generalized confidence interval (GCl), Bayesian methods, the method of variance estimates recovery (MOVER) based on Hendricks and Robey's confidence interval, a percentile bootstrap method, and a bootstrap method with standard errors. To analyze their performances, their coverage probabilities and expected lengths were evaluated via Monte Carlo simulation. The simulation results indicate that the coverage probabilities of $\mathrm{GCl}$ were greater than or sometimes close to the nominal confidence level. However, when the Weibull shape parameter was small, the Bayesian-highest posterior density interval was preferable. All of the proposed confidence intervals were applied to wind speed data measured at 90-meter wind energy potential stations at various regions in Thailand.
\end{abstract}

\title{
INTRODUCTION
}

Nowadays, Thailand is increasingly using alternative energy sources to oil and coal owing to the government pushing forward a policy concerning alternative energy development and efficiency. The alternative energy development in Thailand has primarily relied on energy production in domestic with emphasis on solar, wind, hydro energy, biomass, biogas, municipal Solid waste (MSW), geothermal power and biofuels including ethanol and biodiesel in term of electricity, heat and biofuels (https: //www. dede.go.th/ewt_news.php?nid=47340). Wind energy is an important natural energy source for use in power generation because it has many advantages: it is clean and environmentally friendly, it reduces the level of carbon dioxide emissions that contribute toward global warming, and it offers unlimited renewable energy. In recent years, power generation from wind energy in Thailand has limitation about potential of wind speeds such as wind power density, wind speed, and the height of wind turbine (Chancham et al., 2009). In addition, there are problems about where to install wind turbines because wind energy is related to the topography of the installation area and wind strength variation throughout the year. Potential areas to harvest wind energy in Thailand are coastal areas and the northeastern region of Thailand. Since the coefficient of variation can be used to analyze areas with high wind speed variability, we are interested in analyzing wind datasets from the aforementioned areas in terms of the difference between coefficients of variation of Weibull distributions. The approach in this study could be useful in finding areas that are the most suitable for placing wind turbine systems to produce electricity.

In statistics, the Weibull distribution, which is named after Swedish mathematician Waloddi Weibull (Weibull, 1951), is a continuous probability distribution commonly used to analyze wind speed data. It has also been widely applied in engineering, industry, and weather forecasting. For example, Chaichana et al. 
Let a random variable $X$ follow the 2-Parameter Weibull distribution. The probability density function of $X$ is given by

$$
f(x ; a, k)=\frac{k}{a}\left(\frac{x}{a}\right)^{k-1} \exp \left[-\left(\frac{x}{a}\right)^{k}\right], x>0,
$$

where the positive constant $a$ is called the scale parameter and the positive constant $k$ is called the shape parameter. The mean and the variance of $X$ are $\mathrm{E}(X)=a \Gamma\left(1+\frac{1}{k}\right)$ and $\operatorname{Var}(X)=a^{2}\left[\Gamma\left(1+\frac{2}{k}\right)-\left(\Gamma\left(1+\frac{1}{k}\right)\right)^{2}\right]$,

(2015) studied the potential of wind energy for high-tech agricultural projects as part of the Royal Initiative at Chiang Mai province, Thailand. Moreover, Pang et al. (2001) estimated the parameters for a Weibull distribution when applied to wind speed data obtained from an observatory in Hong Kong. Previously, many researchers have estimated the parameters and functions of parameters for Weibull distributions. For instance, Green et al. (1994) used the Markov chain Monte Carlo (MCMC) method to estimate three parameters of a Weibull distribution. Colosimo and Ho (1999) derived confidence intervals for the Weibull mean lifetime based on censored reliability datasets. Krishnamoorthy et al. (2009) constructed confidence intervals for the mean of Weibull distribution based on the generalized variable approach and compared this with Wald confidence intervals. In another study, Krishnamoorthy and Lin (2010) presented the confidence interval for stress-strength reliability involving two Weibull distributions. Ibrahim (2010) compared the performance of Bayesian approaches using Jeffreys' prior and an extension of Jeffreys' prior with maximum likelihood estimation to estimate the parameters of a Weibull distribution. Kundu and Howlader (2010) used Bayesian inference to estimate the scale parameter of an inverted Weibull distribution based on Type-II censored data. Yalcinkaya and Birgoren (2017) compared a confidence interval of the lower percentiles in a Weibull distribution of small samples based on maximum likelihood estimation and the Bayesian-Weibull method. Saraiva and Suzuki (2017) compared methods to estimate the parameters of a Weibull distribution, which are a maximum likelihood method and a Bayesian method with the Metropolis-Hastings algorithm.

The coefficient of variation is used for measuring variations in data as well as comparing the degree of variation between several datasets in which the measurement units are different. It has been used in many fields. Faber and Korn (1991) used the coefficient of variation to measure variation in the mean synaptic response of nerves in the central nervous system. Banik and Kibria (2011) used the coefficient of variation to analyze data on psychiatric disorders, while Yosboonruang et al. (2019) used it to examine variation in rainfall data in Thailand. In this study, we focus on the coefficient of variation. Many researchers have provided confidence intervals for estimating the single and function of coefficients of variation. For example in a normal distribution: a normal coefficient of variation (Vangel, 1996), common coefficient of variation (Tian, 2005), coefficient of variation (Mahmoudvand and Hassani, 2009) and (Donner and Zou, 2010). Moreover, Pang et al. (2005) proposed confidence intervals for estimating the coefficient of variation of a three-parameter Weibull distribution via a simulation-based Bayesian approach. Niwitpong (2013) provided confidence intervals for the coefficient of variation of a lognormal distribution with a restricted parameter space. Buntao and Niwitpong (2013) constructed confidence intervals for the difference between the coefficients of variation of lognormal distributions. Sangnawakij et al. (2015) presented confidence intervals for the ratio of coefficients of variation of gamma distributions. Yosboonruang et al. (2020) proposed confidence intervals for the difference between the coefficients of variation of delta-lognormal distributions. La-ongkaew et al. (2019) constructed confidence interval for single coefficient of variation of Weibull distribution. It could be seen that not many researches mentioned bove focused on coefficient of variation or difference between the coefficients of variation of Weibull distributions. Consequently, we extended this study to construct confidence intervals for the difference between the coefficients of variation of Weibull distributions.

Herein, we propose new confidence intervals for the difference between the coefficients of variation of Weibull distributions using the generalized confidence interval (GCI) method, Bayesian methods, the method of variance estimates recovery (MOVER) method, a percentile bootstrap method, and a bootstrap interval with standard errors, the derivations of which are given in this article. As an empirical application, we use wind speed data measured at 90-meter wind energy potential stations in Thailand. Finally, we discuss the results of our study and draw conclusions on them.

\section{METHODS}


respectively. Therefore, the coefficient of variation of $X$ can be written as

$$
\mathrm{CV}(X)=\lambda=\sqrt{\frac{\Gamma\left(1+\frac{2}{k}\right)}{\left(\Gamma\left(1+\frac{1}{k}\right)\right)^{2}}-1}
$$

Suppose that $X=\left(X_{1}, X_{2}, \ldots, X_{n}\right)$ be a random sample of size $n$ from Weibull distribution with scale parameter $a$ and shape parameter $k$, denoted as $\operatorname{Weibull}(a, k)$. To estimate the parameters $a$ and $k$, the maximum likelihood estimation is applied. The maximum likelihood estimators (MLEs) can be obtained from Cohen (1965), they are as follows. The MLE $\hat{k}$ of $k$ is solution of the following equation

$$
\frac{1}{\hat{k}}-\frac{\sum_{i=1}^{n}\left[x_{i}^{\hat{k}} \ln \left(x_{i}\right)\right]}{\sum\left(x_{i}^{\hat{k}}\right)}+\frac{1}{n} \sum_{i=1}^{n} \ln \left(x_{i}\right)=0
$$

and the MLE $\hat{a}$ of $a$ is given by

$$
\hat{a}=\left[\sum_{i=1}^{n} x_{i}^{\hat{k}} / n\right]^{\frac{1}{\hat{k}}}
$$

For solving the Eq. (3), the Newton-Raphson iterative method can be applied. We obtained a starting value for the Newton-Raphson iterative method. Let $x_{1}, x_{2}, \ldots, x_{n}$ be a sample of observations from a $\operatorname{Weibull}(a, k)$ distribution. Let $z_{i}=\ln \left(x_{i}\right), i=1,2, \ldots, n$. Menon (1963) showed that the estimator

$$
\hat{k}_{u}=\left(\frac{\pi}{\sqrt{6}}\right)\left(\sqrt{\frac{n-1}{s^{2}}}\right)
$$

Let $X_{1}, X_{2}, \ldots, X_{n}$ and $Y_{1}, Y_{2}, \ldots, Y_{m}$ be random samples from two independent Weibull distributions with scale parameters $a_{X}, a_{Y}$ and shape parameters $k_{X}, k_{Y}$, respectively. Moreover, let $\lambda_{X}$ be the coefficient of variation of $X$. Similarly, let $\lambda_{Y}$ be the coefficient of variation of $Y$. Thus, the difference between the coefficients of variation is defined by

$$
\delta=\lambda_{X}-\lambda_{Y}=\sqrt{\frac{\Gamma\left(1+\frac{2}{k_{X}}\right)}{\left(\Gamma\left(1+\frac{1}{k_{X}}\right)\right)^{2}}-1}-\sqrt{\frac{\Gamma\left(1+\frac{2}{k_{Y}}\right)}{\left(\Gamma\left(1+\frac{1}{k_{Y}}\right)\right)^{2}}-1} .
$$

And the estimator of $\delta$ is given by

$$
\hat{\delta}=\hat{\lambda}_{X}-\hat{\lambda}_{Y}=\sqrt{\frac{\Gamma\left(1+\frac{2}{\hat{k}_{X}}\right)}{\left(\Gamma\left(1+\frac{1}{\hat{k}_{X}}\right)\right)^{2}}-1}-\sqrt{\frac{\Gamma\left(1+\frac{2}{\hat{k}_{Y}}\right)}{\left(\Gamma\left(1+\frac{1}{\hat{k}_{Y}}\right)\right)^{2}}-1 .}
$$




$$
R_{a}=\left(\frac{a}{\hat{a}}\right)^{\frac{\hat{k}}{\hat{k}_{0}}} \hat{a}_{0}=\left(\frac{1}{\hat{a}^{*}}\right)^{\frac{\hat{k}^{*}}{\hat{k}_{0}}} \hat{a}_{0}
$$

From the random variables $X$ and $Y$, the GPQs for $k_{X}$ and $k_{Y}$ are given by

$$
R_{k_{X}}=\frac{\hat{k}_{X_{0}}}{\hat{k}_{X}^{*}}
$$

and

$$
R_{k_{Y}}=\frac{\hat{k}_{Y_{0}}}{\hat{k}_{Y}^{*}}
$$

Thus, the GPQ for $\delta$ is defined by

$$
R_{\delta}=R_{\lambda_{X}}-R_{\lambda_{Y}}=\sqrt{\frac{\Gamma\left(1+\frac{2}{R_{k_{X}}}\right)}{\left(\Gamma\left(1+\frac{1}{R_{k_{X}}}\right)\right)^{2}}-1}-\sqrt{\frac{\Gamma\left(1+\frac{2}{R_{k_{Y}}}\right)}{\left(\Gamma\left(1+\frac{1}{R_{k_{Y}}}\right)\right)^{2}}-1} .
$$

Therefore, the $100(1-\alpha) \%$ two-sided confidence interval for the difference between the coefficients of variation of Weibull distributions based on GCI is

$$
C I_{\delta(g c i)}=\left[L_{\delta(g c i)}, U_{\delta(g c i)}\right]=\left[R_{\delta}(\alpha / 2), R_{\delta}(1-\alpha / 2)\right],
$$

where $R_{\delta}(\alpha / 2)$ denotes the $100(\alpha / 2)$-th percentile of $R_{\delta}$.

The following algorithm is used to construct confidence interval based on GCI for the difference between the coefficients of variation of Weibull distributions: 


\section{Algorithm 2}

1. Compute $\hat{a}_{X}, \hat{a}_{Y}, \hat{k}_{X}$ and $\hat{k}_{Y}$ from Algorithm 1.

2. Generate $X_{1}^{*}, X_{2}^{*}, \ldots, X_{n}^{*}$ and $Y_{1}^{*}, Y_{2}^{*}, \ldots, Y_{m}^{*}$ from $\operatorname{Weibull}(1,1)$.

3. Compute $\hat{a}_{X}^{*}, \hat{a}_{Y}^{*}, \hat{k}_{X}^{*}$ and $\hat{k}_{Y}^{*}$ from Algorithm 1.

4. Compute $R_{k_{X}}$ and $R_{k_{Y}}$ from Eqs. (10) and (11).

5. Compute $R_{\delta}$ from Eq. (12).

6. Repeat steps 1-5 for $q$ times, where $q$ is the number of generalized computation.

7. Compute $95 \%$ confidence interval based on GCI, as given in Eq. (13).

\section{THE BOOTSTRAP CONFIDENCE INTERVALS}

The bootstrap method was introduced by Efron and Tibshirani (1993). This is a resampling method to determine precision measures for statistical estimation.

Let $\mathbf{x}_{i}=\left(x_{1}, x_{2}, \ldots, x_{n}\right)$ and $\mathbf{y}_{i}=\left(y_{1}, y_{2}, \ldots, y_{m}\right)$ be random samples of size $n$ and $m$ from Weibull distributions, and let $\mathbf{x}_{i}^{* b}=\left(x_{1}^{* b}, x_{2}^{* b}, \ldots, x_{n}^{* b}\right)$ and $\mathbf{y}_{i}^{* b}=\left(y_{1}^{* b}, y_{2}^{* b}, \ldots, y_{m}^{* b}\right)$ be bootstrapped samples drawn with replacement from the original data, using the same sample sizes. After resampling $B$ bootstrap samples, the difference between the coefficients of variation are calculated in each bootstrap sample, as follows: $\delta^{* b}=\lambda_{X}^{* b}-\lambda_{Y}^{* b}, b=1,2, \ldots, B$.

\section{The percentile bootstrap confidence interval}

The percentile bootstrap confidence interval is based on the percentile of the distribution of the bootstrapped replications. The value of the bootstrap statistic, $\delta^{* b}$ are ordered from the smallest to the largest.

Therefore, the $100(1-\alpha) \%$ two-sided confidence interval for the difference between the coefficients of variation of Weibull distributions based on percentile bootstrap is given by

$$
C I_{\delta(p b)}=\left[L_{\delta(p b)}, U_{\delta(p b)}\right]=\left[\delta^{* b}(\alpha / 2), \delta^{* b}(1-\alpha / 2)\right],
$$

where $\delta^{* b}(\alpha / 2)$ denotes the $100(\alpha / 2)$-th percentile of $\delta^{* b}$.

The following algorithm is used to construct confidence interval based on percentile bootstrap for the difference between the coefficients of variation of Weibull distributions:

\section{Algorithm 3}

1. Generate $X_{1}, X_{2}, \ldots, X_{n}$ from Weibull $\left(a_{X}, k_{X}\right)$ and $Y_{1}, Y_{2}, \ldots, Y_{m}$ from Weibull $\left(a_{Y}, k_{Y}\right)$.

2. For $b=1$.

3. Resampling bootstrap samples $X_{1}^{*}, X_{2}^{*}, \ldots, X_{n}^{*}$ from $X_{1}, X_{2}, \ldots, X_{n}$ and compute $\lambda_{X}^{* b}$.

4. Resampling bootstrap samples $Y_{1}^{*}, Y_{2}^{*}, \ldots, Y_{m}^{*}$ from $Y_{1}, Y_{2}, \ldots, Y_{m}$ and compute $\lambda_{Y}^{* b}$.

5. Compute $\delta^{* b}=\lambda_{X}^{* b}-\lambda_{Y}^{* b}$.

6. Repeat steps 3-5 for $B$ times, where $B$ is the number of bootstrap sample.

7. Sort $\delta^{* b}$ from the smallest to the largest.

8. Compute 95\% confidence interval based on percentile bootstrap, as given in Eq. (14).

\section{The bootstrap confidence interval with standard errors}

From the $B$ bootstrap statistic denoted as $\delta^{* b}, b=1,2, \ldots, B$, we can calculate the standard error of a statistic. They can be estimated using the standard deviation of the bootstrap distribution.

Therefore, the $100(1-\alpha) \%$ two-sided confidence interval for the difference between the coefficients of variation of Weibull distributions based on bootstrap confidence interval with standard errors can be written as

$$
C I_{\delta(b s)}=\left[L_{\delta(b s)}, U_{\delta(b s)}\right]=\left[\hat{\delta}-Z_{(\alpha / 2)} S E, \hat{\delta}+Z_{(\alpha / 2)} S E\right],
$$

where $S E$ is the error of a statistic.

The following algorithm is used to construct confidence interval based on bootstrap confidence interval with standard errors for the difference between the coefficients of variation of Weibull distributions:

Algorithm 4 
1. Generate $X_{1}, X_{2}, \ldots, X_{n}$ from Weibull $\left(a_{X}, k_{X}\right)$ and $Y_{1}, Y_{2}, \ldots, Y_{m}$ from Weibull $\left(a_{Y}, k_{Y}\right)$.

2. For $b=1$.

3. Resampling bootstrap samples $X_{1}^{*}, X_{2}^{*}, \ldots, X_{n}^{*}$ from $X_{1}, X_{2}, \ldots, X_{n}$ and compute $\lambda_{X}^{* b}$.

4. Resampling bootstrap samples $Y_{1}^{*}, Y_{2}^{*}, \ldots, Y_{m}^{*}$ from $Y_{1}, Y_{2}, \ldots, Y_{m}$ and compute $\lambda_{Y}^{* b}$.

5. Compute $\delta^{* b}=\lambda_{X}^{* b}-\lambda_{Y}^{* b}$.

6. Repeat steps 3-5 for $B$ times, where $B$ is the number of bootstrap sample.

7. Compute $S E$.

8. Compute $95 \%$ confidence interval based on bootstrap confidence interval with standard errors, as given in Eq. (15).

\section{THE METHOD OF VARIANCE ESTIMATES RECOVERY}

According to Donner and Zou (2010), this approach can be used to construct a confidence interval for a function of two parameters, $\lambda_{X}-\lambda_{Y}$, defined as

$$
C I_{m}=\left[L_{m}, U_{m}\right],
$$

where the lower limit and upper limit for $\hat{\lambda}_{X}-\hat{\lambda}_{Y}$ are given by

$$
\begin{gathered}
L_{m}=\left(\hat{\lambda}_{X}-\hat{\lambda}_{Y}\right)-\sqrt{\left(\hat{\lambda}_{X}-l_{X}\right)^{2}+\left(u_{Y}-\hat{\lambda}_{Y}\right)^{2}} \\
U_{m}=\left(\hat{\lambda}_{X}-\hat{\lambda}_{Y}\right)+\sqrt{\left(u_{X}-\hat{\lambda}_{X}\right)^{2}+\left(\hat{\lambda}_{Y}-l_{Y}\right)^{2}} .
\end{gathered}
$$

Hendricks and Robey (1936) presented confidence intervals for $\lambda_{X}$ and $\lambda_{Y}$ defined as

$$
\left(l_{X . H R}, u_{X . H R}\right)=\left(\hat{\lambda}_{X}-t_{(\alpha / 2, n-1)} \frac{\hat{\lambda}_{X}}{\sqrt{2 n}}, \hat{\lambda}_{X}+t_{(\alpha / 2, n-1)} \frac{\hat{\lambda}_{X}}{\sqrt{2 n}}\right)
$$

and

$$
\left(l_{Y . H R}, u_{Y . H R}\right)=\left(\hat{\lambda}_{Y}-t_{(\alpha / 2, m-1)} \frac{\hat{\lambda}_{Y}}{\sqrt{2 m}}, \hat{\lambda}_{Y}+t_{(\alpha / 2, m-1)} \frac{\hat{\lambda}_{Y}}{\sqrt{2 m}}\right),
$$

where $t_{(\alpha / 2, n-1)}$ and $t_{(\alpha / 2, m-1)}$ denote the $100(\alpha / 2)$-th percentile of t-distribution with $n-1$ and $m-1$ degrees of freedom, respectively. To construct a confidence interval for the difference between the coefficients of variation of Weibull distributions based on MOVER with Hendricks and Robey's confidence interval, we substitute $l_{X . H R}, u_{X . H R}, l_{Y . H R}$ and $u_{Y . H R}$ for $l_{X}, u_{X}, l_{Y}$ and $u_{Y}$, respectively.

Therefore, the $100(1-\alpha) \%$ two-sided confidence interval for the difference between the coefficients of variation of Weibull distributions based on MOVER with Hendricks and Robey's confidence interval becomes

$$
C I_{\delta(m . H R)}=\left[L_{\delta(m . H R)}, U_{\delta(m . H R)}\right],
$$

where

$$
\begin{gathered}
L_{\delta(m . H R)}=\left(\hat{\lambda}_{X}-\hat{\lambda}_{Y}\right)-\sqrt{\left(\hat{\lambda}_{X}-l_{X . H R}\right)^{2}+\left(u_{Y . H R}-\hat{\lambda}_{Y}\right)^{2}} \\
U_{\delta(m . H R)}=\left(\hat{\lambda}_{X}-\hat{\lambda}_{Y}\right)+\sqrt{\left(u_{X . H R}-\hat{\lambda}_{X}\right)^{2}+\left(\hat{\lambda}_{Y}-l_{Y . H R}\right)^{2}} .
\end{gathered}
$$

The following algorithm is used to construct confidence interval based on MOVER for the difference between the coefficients of variation of Weibull distributions:

\section{Algorithm 5}

1. Generate $X_{1}, X_{2}, \ldots, X_{n}$ from Weibull $\left(a_{X}, k_{X}\right)$ and $Y_{1}, Y_{2}, \ldots, Y_{m}$ from Weibull $\left(a_{Y}, k_{Y}\right)$.

2. Compute the intervals for $\lambda_{X}$ and $\lambda_{Y}$ from Eqs. (19) and (20).

3. Compute $95 \%$ confidence interval based on MOVER, as given in Eq. (21). 
Here, we derive Bayesian estimates of the parameters of a Weibull distribution. Once again, for random variable $\mathrm{X}=\left(X_{1}, X_{2}, \ldots, X_{n}\right)$ from a Weibull distribution, we transform scale parameter $a^{\prime}=\left(\frac{1}{a}\right)^{k}$, and so we can provide its probability density function in another form as follows:

$$
f\left(x ; a^{\prime}, k\right)=a^{\prime} k x^{k-1} \exp \left[a^{\prime} x^{k}\right], x>0 .
$$

It is assumed that the prior distributions for the scale and shape parameters are gamma with hyperparameters $\left(v_{1}, v_{2}, z_{1}, z_{2}\right)$ :

$$
\pi\left(a^{\prime}\right) \sim \operatorname{gamma}\left(v_{1}, z_{1}\right)
$$

and

$$
\pi(k) \sim \operatorname{gamma}\left(v_{2}, z_{2}\right) .
$$

Hence, using Bayes' theorem, the joint posterior density function of $a^{\prime}$ and $k$ is given by

$$
\pi\left(a^{\prime}, k \mid x\right) \propto L\left(a^{\prime}, k \mid x\right) \pi\left(a^{\prime}\right) \pi(k),
$$

where $L\left(a^{\prime}, k \mid x\right)$ is a likelihood function.

However, the posterior distributions cannot be computed, and so we used the MCMC method with Gibbs sampling to provide them for the parameters. We used the Gibbs sampling procedure to generate samples from the joint posterior distribution, as given in Eq. (27). The conditional posterior distributions of parameters are

$$
\pi\left(a^{\prime} \mid k, x\right) \sim \operatorname{gamma}\left(v_{2}+n, z_{2}+\sum x^{k}\right)
$$

and

$$
\pi\left(k \mid a^{\prime}, x\right) \propto k^{v_{1}+n-1} \exp \left[-v_{1} k-a^{\prime} \sum x^{k}\right] .
$$

For Eq. (29), we used the Metropolis-Hasting (MH) algorithm to update shape parameter $k$. Moreover, the random walk Metropolis (RWM) and Gibbs sampling methods are applied as follows (Geman and Geman, 1984):

Algorithm 6 The Gibbs algorithm

1. Take the initial value of parameter $\left(a^{\prime(0)}, k^{(0)}\right)$.

2. Generate $a^{\prime(t)} \sim \operatorname{gamma}\left(v_{2}+n, z_{2}+\sum x^{k^{(t-1)}}\right)$.

3. Using Random walk Metropolis (RWM) algorithm for calculate $k^{(t)}$.

4. Repeat step 2-3 for $T$ times, where $T$ is the number of replications of MCMC.

5. Burn in 1000 samples and compute the parameter of interest.

Algorithm 7 Random walk Metropolis (RWM)

1. Start with $\left(a^{\prime(t)}, k^{(t-1)}\right)$.

2. Generate $\varepsilon \sim N\left(0, \sigma_{k}^{2}\right)$.

3. Compute $k^{*}=k^{(t-1)}+\varepsilon$.

4. Compute $A_{k}=\frac{L\left(k^{*}, a^{\prime} \mid x\right) \pi\left(k^{*}\right)}{L\left(k, a^{\prime} \mid x\right) \pi(k)}$.

5. Generate $u \sim U(0,1)$.

6. If $u \leq \min \left(1, A_{k}\right)$ set $k^{(t)}=k^{*}$ and If $u>\min \left(1, A_{k}\right)$ set $k^{(t)}=k^{(t-1)}$.

\section{The Bayesian-MCMC}

Once again, let $X$ and $Y$ be random variables from Weibull distributions. First, we used Algorithms 6 and 7 to calculate the difference between the coefficients of variation denoted as $\delta^{t}, t=1,2, \ldots, T$, based on the Bayesian-MCMC. 
Therefore, the $100(1-\alpha) \%$ two-sided confidence interval for the difference between the coefficients of variation of Weibull distributions based on the Bayesian-MCMC is given by

$$
C I_{\delta(M C M C)}=\left[L_{\delta(M C M C)}, U_{\delta(M C M C)}\right]=\left[\delta^{t}(\alpha / 2), \delta^{t}(1-\alpha / 2)\right],
$$

where $\delta^{t}(\alpha / 2)$ denotes the $100(\alpha / 2)$-th percentile of $\delta^{t}$.

The following algorithm is used to construct confidence interval based on Bayesian-MCMC for the difference between the coefficients of variation of Weibull distributions:

\section{Algorithm 8}

1. Calculate the initial value of parameter $\left(a^{\prime(0)}, k^{(0)}\right)$.

2. Calculate $\delta^{t}$ from Algorithms 6 and 7.

3. Compute $95 \%$ confidence interval based on Bayesian-MCMC, as given in Eq. (30).

\section{The Bayesian-Highest Posterior Density (HPD) Interval}

Here, the assumption is that the density of every point inside the interval is greater than that of every point outside the interval, and it is also the shortest interval (Box and Tiao, 2011). In this article, the HPD interval was computed by using the R package with HDIInterval.

Therefore, the $100(1-\alpha) \%$ two-sided confidence interval for the difference between the coefficients of variation of Weibull distributions based on the Bayesian-HPD interval can be written as

$$
C I_{\delta(H P D)}=\left[L_{\delta(H P D)}, U_{\delta(H P D)}\right] .
$$

The following algorithm is used to construct confidence interval based on Bayesian-HPD for the difference between the coefficients of variation of Weibull distributions:

\section{Algorithm 9}

1. Calculate the initial value of parameter $\left(a^{(0)}, k^{(0)}\right)$.

2. Calculate $\delta^{t}$ from Algorithms 6 and 7.

3. Compute $95 \%$ confidence interval based on Bayesian-HPD interval, as given in Eq. (31).

\section{RESULTS}

A simulation study was conducted to compare the performances of the six confidence intervals for the difference between the coefficients of variation of Weibull distributions: GCI, the Bayesian-MCMC method, the Bayesian-HPD interval, MOVER based on Hendricks and Robey's confidence interval, the percentile bootstrap method, and the bootstrap confidence interval with standard errors. The $\mathrm{R}$ program was used to estimate the coverage probabilities and expected lengths of the proposed confidence intervals using the following parameter settings:

Scale parameter $a_{X}=a_{y}=0.5$ and 2 .

Shape parameter $k_{X}=1$ and $k_{Y}=0,1,2,2.5,4$ and 9 , with the differences between the coefficients of variation of $-1.2360,0,0.4772,0.5720,0.7194$ and 0.8671 , respectively.

Sample sizes $(n, m)=(10,10),(10,20),(20,20),(30,30),(30,50),(50,50),(50,100)$ and $(100,100)$.

Hyperparameters $v_{1}=v_{2}=z_{1}=z_{2}=0.1$.

The number of replications for each situation was 5,000, along with 2,500 pivotal quantities for GCI. Moreover, we used 500 bootstrap samples for the bootstrap methods and we generated 20,000 realizations of MCMC using the Gibbs and RWM algorithms with a burn-in of 1000. The following performance indicators were used to determine the best-performing method: a coverage probability of greater than or equal to 0.95 (the nominal confidence level) and the shortest expected length.

The following algorithm is used to estimate the coverage probability and expected length:

\section{Algorithm 10}

1. Set $M, q, B, n, m, a_{X}, k_{X}, a_{Y}$ and $k_{Y}$.

2. Generate $X_{1}, X_{2}, \ldots, X_{n}$ from Weibull $\left(a_{X}, k_{X}\right)$ and $Y_{1}, Y_{2}, \ldots, Y_{m}$ from Weibull $\left(a_{Y}, k_{Y}\right)$, respectively.

3. Use Algorithm 2 to construct generalized confidence interval $\left(C I_{\delta(g c i)}\right)$.

4. Use Algorithm 3 to construct percentile bootstrap confidence interval $\left(C I_{\delta(p b)}\right)$.

5. Use Algorithm 4 to construct bootstrap confidence interval with standard errors $\left(C I_{\delta(b s)}\right)$. 
6. Use Algorithm 5 to construct MOVER based on Hendricks and Robey's confidence interval $\left(C I_{\delta(m . H R)}\right)$.

7. Use Algorithm 8 to construct Baysian-MCMC confidence interval $\left(C I_{\delta(M C M C)}\right)$.

8. Use Algorithm 9 to construct Baysian-HPD confidence interval $\left(C I_{\delta(H P D)}\right)$.

9. If $(L \leq \delta \leq U)$, then set $P=1$, else set $P=0$.

10. Compute $(U-L)$.

11. Repeat steps 2-10 for $M$ times.

12. Compute mean of $P$ for the coverage probability.

13. Compute mean of $(U-L)$ for the expected length.

For $a=0.5$ (Table 1), the simulation results indicate that the coverage probabilities of $C I_{\delta(g c i)}$ were greater than or sometimes close to the nominal confidence level of 0.95 in almost all cases. $C I_{\delta(p b)}$ yielded coverage probabilities greater than 0.95 when $k_{y}=1$ for $(n, m)=(10,10)$ and $(20,20)$. For the Bayesian methods, the coverage probabilities of $C I_{\delta(M C M C)}$ and $C I_{\delta(H P D)}$ were greater than or sometimes close to the nominal confidence level, while the expected lengths of $C I_{\delta(H P D)}$ for $(n, m)=(10,10)$ and $k_{y}=0.5,2,4 ;(n, m)=(20,20)$ and $k_{y}=0.5 ;(n, m)=(10,20),(30,30),(30,50),(50,50),(50,100)$ and $k_{y}=0.5,1 ;$ and $(n, m)=(100,100)$ and $k_{y}=1$ were the shortest. Moreover, $C I_{\delta(b s)}$ and $C I_{\delta(m . H R)}$ yielded coverage probabilities under 0.95 for all cases. From the simulation results for $a=2$ (Table 2), $C I_{\delta(g c i)}$ performed well in terms of coverage probability in almost all cases, while those of $C I_{\delta(p b)}, C I_{\delta(b s)}$ and $C I_{\delta(m . H R)}$ were the same as for $a=0.5 . C I_{\delta(p b)}$ yielded coverage probabilities of under 0.95 in almost all cases except when $(n, m)=(10,10),(20,20)$ and $k_{y}=0.5$. For the Bayesian methods, the coverage probabilities of $C I_{\delta(H P D)}$ were greater than 0.95 and its expected lengths were shortest for $(n, m)=(10,20),(30,50),(50,100)$ and $k_{y}=0.5,1 ;(n, m)=(20,20)$ and $k_{y}=2 ;(n, m)=(30,30)$ and $k_{y}=1,2,2.5 ;(n, m)=(50,50)$ and $k_{y}=0.5,1,2.5 ;$ and $(n, m)=(100,100)$ for all $k_{y}$ except for $k_{y}=4$ and 9 . Finally, the coverage probability and expected length are considered with varying the difference between coefficients of variation and sample size, which can be seen in Figures 1 and 2.
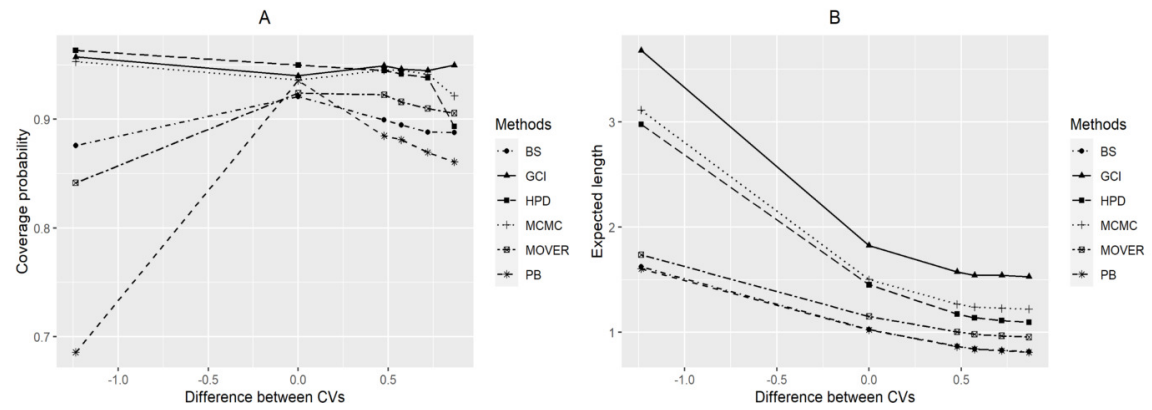

Figure 1. Graphs to compare the performance of the methods with varying difference between CVs in terms of (A) Coverage probability (B) Expected length.
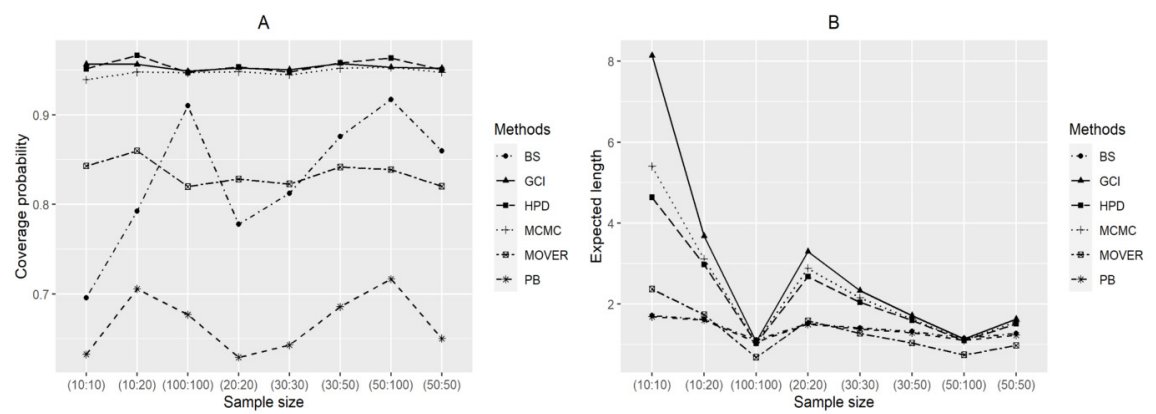

Figure 2. Graphs to compare the performance of the methods with varying sample size in terms of (A) Coverage probability (B) Expected length. 
Table 1. Comparison results of the $95 \%$ two-sided confidence intervals for the difference between the coefficients of variation of Weibull distributions for $a=0.5$.

\begin{tabular}{|c|c|c|c|c|c|c|c|}
\hline \multirow{2}{*}{$(n, m)$} & \multirow{2}{*}{$k$} & \multicolumn{6}{|c|}{ Coverage probability (Expected length) } \\
\hline & & $C I_{\delta(g c i)}$ & $C I_{\delta(p b)}$ & $C I_{\delta(b s)}$ & $C I_{\delta(m . H R)}$ & $C I_{\delta(M C M C)}$ & $C I_{\delta(H P D)}$ \\
\hline \multirow[t]{12}{*}{$(10,10)$} & \multirow[t]{2}{*}{0.5} & 0.9568 & 0.6324 & 0.6956 & 0.8430 & 0.9392 & 0.9514 \\
\hline & & $(8.1452)$ & $(1.6874)$ & $(1.7114)$ & $(2.3678)$ & $(5.4003)$ & (4.6368) \\
\hline & \multirow[t]{2}{*}{1} & 0.9494 & 0.9614 & 0.9032 & 0.9610 & 0.9388 & 0.9706 \\
\hline & & (2.3498) & (1.1568) & (1.1587) & $(1.3535)$ & $(1.8406)$ & (1.7986) \\
\hline & \multirow[t]{2}{*}{2} & 0.9510 & 0.9046 & 0.8860 & 0.9410 & 0.9454 & 0.9578 \\
\hline & & (1.7089) & $(0.9219)$ & $(0.9248)$ & $(1.0787)$ & (1.3696) & (1.3018) \\
\hline & \multirow[t]{2}{*}{2.5} & 0.9492 & 0.8710 & 0.8610 & 0.9152 & 0.9370 & 0.9400 \\
\hline & & (1.6386) & $(0.8863)$ & $(0.8894)$ & $(1.0359)$ & $(1.3144)$ & $(1.2340)$ \\
\hline & \multirow[t]{2}{*}{4} & 0.9580 & 0.8604 & 0.8526 & 0.9026 & 0.9360 & 0.9510 \\
\hline & & $(1.5526)$ & $(0.8366)$ & $(0.8404)$ & $(0.9827)$ & $(1.2451)$ & (1.1469) \\
\hline & \multirow[t]{2}{*}{9} & 0.9510 & 0.8374 & 0.8334 & 0.8854 & 0.9120 & 0.8764 \\
\hline & & (1.5173) & $(0.8076)$ & $(0.8105)$ & $(0.9515)$ & (1.2218) & $(1.1060)$ \\
\hline \multirow[t]{12}{*}{$(10,20)$} & \multirow[t]{2}{*}{0.5} & 0.9564 & 0.7056 & 0.7924 & 0.8598 & 0.9476 & 0.9664 \\
\hline & & $(3.6785)$ & $(1.6034)$ & $(1.6216)$ & $(1.7373)$ & $(3.1102)$ & ( 2.9756$)$ \\
\hline & \multirow[t]{2}{*}{1} & 0.9504 & 0.9378 & 0.8948 & 0.9344 & 0.9416 & 0.9616 \\
\hline & & $(1.8251)$ & $(1.0235)$ & (1.0258) & (1.1510) & (1.5018) & (1.4525) \\
\hline & \multirow[t]{2}{*}{2} & 0.9536 & 0.8620 & 0.8536 & 0.9018 & 0.9406 & 0.9362 \\
\hline & & (1.5737) & $(0.8642)$ & $(0.8664)$ & (1.0037) & $(1.2677)$ & (1.1743) \\
\hline & \multirow[t]{2}{*}{2.5} & 0.9543 & 0.8480 & 0.8448 & 0.8952 & 0.9382 & 0.9300 \\
\hline & & (1.5418) & $(0.8407)$ & $(0.8432)$ & $(0.9810)$ & $(1.2400)$ & (1.1376) \\
\hline & \multirow[t]{2}{*}{4} & 0.9488 & 0.8380 & 0.8312 & 0.8836 & 0.9386 & 0.9154 \\
\hline & & (1.5419) & $(0.8264)$ & $(0.8302)$ & $(0.9668)$ & $(1.2290)$ & (1.1124) \\
\hline & \multirow[t]{2}{*}{9} & 0.9482 & 0.8262 & 0.8250 & 0.8786 & 0.9204 & 0.8794 \\
\hline & & $(1.5300)$ & $(0.8106)$ & $(0.8144)$ & $(0.9544)$ & (1.2208) & $(1.0958)$ \\
\hline \multirow[t]{12}{*}{$(20,20)$} & \multirow[t]{2}{*}{0.5} & 0.9524 & 0.6292 & 0.7778 & 0.8282 & 0.9480 & 0.9536 \\
\hline & & (3.2987) & (1.4974) & $(1.5220)$ & $(1.5855)$ & $(2.8839)$ & (2.6795) \\
\hline & \multirow[t]{2}{*}{1} & 0.9530 & 0.9564 & 0.9170 & 0.9450 & 0.9444 & 0.9656 \\
\hline & & $(1.2350)$ & $(0.8758)$ & $(0.8814)$ & $(0.9149)$ & (1.1264) & (1.1166) \\
\hline & 2 & 0.9522 & 0.9024 & 0.9010 & 0.9330 & 0.9434 & 0.9486 \\
\hline & & $(0.9234)$ & $(0.6836)$ & $(0.6889)$ & $(0.7236)$ & $(0.8471)$ & $(0.8260)$ \\
\hline & 2.5 & 0.9526 & 0.8900 & 0.8918 & 0.9276 & 0.9458 & 0.9470 \\
\hline & & $(0.8915)$ & $(0.6566)$ & $(0.6615)$ & $(0.6969)$ & $(0.8178)$ & $(0.7917)$ \\
\hline & 4 & 0.9534 & 0.8730 & 0.8870 & 0.9166 & 0.9430 & 0.9344 \\
\hline & & $(0.8589)$ & $(0.6348)$ & $(0.6403)$ & $(0.6666)$ & $(0.7866)$ & $(0.7532)$ \\
\hline & 9 & 0.9522 & 0.8652 & 0.8720 & 0.9114 & 0.9194 & 0.8866 \\
\hline & & $(0.8395)$ & $(0.6149)$ & $(0.6225)$ & $(0.6479)$ & $(0.7729)$ & $(0.7340)$ \\
\hline$(30,30)$ & 0.5 & 0.9506 & 0.6428 & 0.8124 & 0.8228 & 0.9448 & 0.9501 \\
\hline & & (2.3298) & $(1.3805)$ & (1.4109) & $(1.2717)$ & (2.1510) & (2.0452) \\
\hline & 1 & 0.9522 & 0.9426 & 0.9244 & 0.9382 & 0.9480 & 0.9618 \\
\hline & & $(0.9229)$ & $(0.7415)$ & $(0.7481)$ & $(0.7346)$ & $(0.8728)$ & $(0.8678)$ \\
\hline & 2 & 0.9510 & 0.8972 & 0.9082 & 0.9298 & 0.9462 & 0.9482 \\
\hline & & $(0.6990)$ & $(0.5770)$ & $(0.5819)$ & $(0.5820)$ & $(0.6621)$ & $(0.6506)$ \\
\hline & 2.5 & 0.9482 & 0.8918 & 0.9064 & 0.9280 & 0.9452 & 0.9476 \\
\hline & & $(0.6787)$ & $(0.5581)$ & $(0.5629)$ & $(0.5625)$ & $(0.6430)$ & $(0.6287)$ \\
\hline & 4 & 0.9532 & 0.8776 & 0.9036 & 0.9212 & 0.9476 & 0.9394 \\
\hline & & $(0.6526)$ & $(0.5364)$ & $(0.5429)$ & $(0.5370)$ & $(0.6171)$ & $(0.5991)$ \\
\hline & 9 & 0.9446 & 0.8602 & 0.8814 & 0.9036 & 0.9080 & 0.8784 \\
\hline & & $(0.6365)$ & $(0.5187)$ & $(0.5246)$ & $(0.5207)$ & $(0.6040)$ & $(0.5835)$ \\
\hline
\end{tabular}


Table 1 (Continued)

\begin{tabular}{|c|c|c|c|c|c|c|c|}
\hline \multirow{2}{*}{$(n, m)$} & \multirow{2}{*}{$k$} & \multicolumn{6}{|c|}{ Coverage probability (Expected length) } \\
\hline & & $C I_{\delta(g c i)}$ & $C I_{\delta(p b)}$ & $C I_{\delta(b s)}$ & $C I_{\delta(m . H R)}$ & $C I_{\delta(M C M C)}$ & $C I_{\delta(H P D)}$ \\
\hline \multirow[t]{12}{*}{$(30,50)$} & \multirow[t]{2}{*}{0.5} & 0.9574 & 0.6856 & 0.8760 & 0.8416 & 0.9530 & 0.9634 \\
\hline & & $(1.7122)$ & $(1.2865)$ & $(1.3262)$ & $(1.0347)$ & $(1.6309)$ & (1.5927) \\
\hline & \multirow[t]{2}{*}{1} & 0.9400 & 0.9356 & 0.9210 & 0.9240 & 0.9360 & 0.9500 \\
\hline & & $(0.8011)$ & $(0.6768)$ & $(0.6822)$ & $(0.6526)$ & $(0.7652)$ & $(0.7591)$ \\
\hline & \multirow[t]{2}{*}{2} & 0.9494 & 0.8848 & 0.8996 & 0.9226 & 0.9452 & 0.9448 \\
\hline & & $(0.6690)$ & $(0.5518)$ & $(0.5568)$ & $(0.5556)$ & $(0.6354)$ & $(0.6203)$ \\
\hline & \multirow[t]{2}{*}{2.5} & 0.9462 & 0.8812 & 0.8948 & 0.9160 & 0.9452 & 0.9418 \\
\hline & & $(0.6581)$ & $(0.5399)$ & $(0.5455)$ & $(0.5440)$ & $(0.6246)$ & $(0.6074)$ \\
\hline & \multirow[t]{2}{*}{4} & 0.9450 & 0.8696 & 0.8880 & 0.9098 & 0.9414 & 0.9382 \\
\hline & & $(0.6447)$ & $(0.5297)$ & $(0.5359)$ & $(0.5289)$ & $(0.6095)$ & $(0.5897)$ \\
\hline & \multirow[t]{2}{*}{9} & 0.9498 & 0.8608 & 0.8878 & 0.9056 & 0.9214 & 0.8936 \\
\hline & & $(0.6306)$ & $(0.5144)$ & $(0.5208)$ & $(0.5170)$ & $(0.5981)$ & $(0.5769)$ \\
\hline \multirow[t]{12}{*}{$(50,50)$} & \multirow[t]{2}{*}{0.5} & 0.9520 & 0.6502 & 0.8596 & 0.8202 & 0.9474 & 0.9504 \\
\hline & & $(1.6289)$ & $(1.2294)$ & $(1.2703)$ & $(0.9739)$ & $(1.5586)$ & (1.5103) \\
\hline & \multirow[t]{2}{*}{1} & 0.9474 & 0.9368 & 0.9330 & 0.9278 & 0.9456 & 0.9552 \\
\hline & & $(0.6699)$ & $(0.6106)$ & $(0.6165)$ & $(0.5626)$ & $(0.6485)$ & $(0.6460)$ \\
\hline & \multirow[t]{2}{*}{2} & 0.9456 & 0.9058 & 0.9208 & 0.9304 & 0.9460 & 0.9480 \\
\hline & & $(0.5171)$ & $(0.4724)$ & $(0.4766)$ & $(0.4489)$ & $(0.5010)$ & $(0.4951)$ \\
\hline & \multirow[t]{2}{*}{2.5} & 0.9512 & 0.8900 & 0.9178 & 0.9264 & 0.9448 & 0.9450 \\
\hline & & $(0.4992)$ & $(0.4545)$ & $(0.4586)$ & $(0.4319)$ & $(0.4840)$ & $(0.4770)$ \\
\hline & \multirow[t]{2}{*}{4} & 0.9558 & 0.8844 & 0.9154 & 0.9228 & 0.9482 & 0.9412 \\
\hline & & $(0.4796)$ & $(0.4384)$ & $(0.4432)$ & $(0.4119)$ & $(0.4640)$ & $(0.4552)$ \\
\hline & \multirow[t]{2}{*}{9} & 0.9458 & 0.8744 & 0.9082 & 0.9148 & 0.9132 & 0.8908 \\
\hline & & $(0.4695)$ & $(0.4246)$ & $(0.4299)$ & $(0.4008)$ & $(0.4564)$ & $(0.4463)$ \\
\hline \multirow[t]{12}{*}{$(50,100)$} & \multirow[t]{2}{*}{0.5} & 0.9532 & 0.7166 & 0.9172 & 0.8386 & 0.9518 & 0.9580 \\
\hline & & (1.1384) & $(1.1032)$ & (1.1514) & $(0.7422)$ & (1.1119) & (1.0987) \\
\hline & \multirow[t]{2}{*}{1} & 0.9584 & 0.9364 & 0.9448 & 0.9346 & 0.9538 & 0.9610 \\
\hline & & $(0.5672)$ & $(0.5404)$ & $(0.5448)$ & $(0.4843)$ & $(0.5522)$ & $(0.5486)$ \\
\hline & 2 & 0.9480 & 0.8898 & 0.9176 & 0.9244 & 0.9460 & 0.9484 \\
\hline & & $(0.4897)$ & $(0.4464)$ & $(0.4505)$ & $(0.4230)$ & $(0.4759)$ & $(0.4684)$ \\
\hline & 2.5 & 0.9518 & 0.8820 & 0.9098 & 0.9116 & 0.9496 & 0.9442 \\
\hline & & $(0.4814)$ & $(0.4380)$ & $(0.4425)$ & $(0.4145)$ & $(0.4668)$ & $(0.4581)$ \\
\hline & 4 & 0.9494 & 0.8734 & 0.9082 & 0.9164 & 0.9466 & 0.9418 \\
\hline & & $(0.4700)$ & $(0.4309)$ & $(0.4361)$ & $(0.4041)$ & $(0.4567)$ & $(0.4471)$ \\
\hline & 9 & 0.9486 & 0.8704 & 0.9006 & 0.9074 & - & - \\
\hline & & $(0.4648)$ & $(0.4206)$ & $(0.4253)$ & $(0.3974)$ & - & - \\
\hline$(100,100)$ & 0.5 & 0.9488 & 0.6768 & 0.9102 & 0.8198 & 0.9468 & 0.9472 \\
\hline & & $(1.0705)$ & $(1.0617)$ & (1.1132) & $(0.6829)$ & $(1.0486)$ & (1.0308) \\
\hline & 1 & 0.9496 & 0.9362 & 0.9548 & 0.9266 & 0.9496 & 0.9524 \\
\hline & & $(0.4523)$ & $(0.4660)$ & $(0.4692)$ & $(0.3949)$ & $(0.4453)$ & $(0.4440)$ \\
\hline & 2 & 0.9434 & 0.9076 & 0.9346 & 0.9256 & 0.9444 & 0.9460 \\
\hline & & $(0.3514)$ & $(0.3558)$ & $(0.3584)$ & $(0.3152)$ & $(0.3461)$ & $(0.3437)$ \\
\hline & 2.5 & 0.9514 & 0.9060 & 0.9386 & 0.9286 & 0.9486 & 0.9492 \\
\hline & & $(0.3394)$ & $(0.3435)$ & $(0.3462)$ & $(0.3032)$ & $(0.3341)$ & $(0.3313)$ \\
\hline & 4 & 0.9532 & 0.9004 & 0.9390 & 0.9270 & 0.9516 & 0.9484 \\
\hline & & $(0.3269)$ & $(0.3344)$ & $(0.3376)$ & $(0.2896)$ & $(0.3214)$ & $(0.3179)$ \\
\hline & 9 & 0.9514 & 0.8954 & 0.9336 & 0.9224 & - & - \\
\hline & & $(\mathbf{0 . 3 1 8 8})$ & $(0.3223)$ & $(0.3250)$ & $(0.2809)$ & - & - \\
\hline
\end{tabular}


Table 2. Comparison results of the 95\% two-sided confidence intervals for the difference between the coefficients of variation of Weibull distributions for $a=2$.

\begin{tabular}{|c|c|c|c|c|c|c|c|}
\hline \multirow{2}{*}{$(n, m)$} & \multirow{2}{*}{$k$} & \multicolumn{6}{|c|}{ Coverage probability (Expected length) } \\
\hline & & $C I_{\delta(g c i)}$ & $C I_{\delta(p b)}$ & $C I_{\delta(b s)}$ & $C I_{\delta(m . H R)}$ & $C I_{\delta(M C M C)}$ & $C I_{\delta(H P D)}$ \\
\hline \multirow[t]{12}{*}{$(10,10)$} & \multirow[t]{2}{*}{0.5} & 0.9512 & 0.6242 & 0.6852 & 0.8266 & 0.9368 & 0.9466 \\
\hline & & (7.9720) & $(1.6763)$ & $(1.6970)$ & $(2.3414)$ & $(5.2853)$ & $(4.5489)$ \\
\hline & \multirow[t]{2}{*}{1} & 0.9514 & 0.9630 & 0.9034 & 0.9632 & 0.9338 & 0.9674 \\
\hline & & $(2.3568)$ & (1.1613) & $(1.1622)$ & $(1.3561)$ & $(1.8368)$ & $(1.7938)$ \\
\hline & \multirow[t]{2}{*}{2} & 0.9430 & 0.8992 & 0.8744 & 0.9330 & 0.9300 & 0.9482 \\
\hline & & $(1.7036)$ & $(0.9226)$ & $(0.9261)$ & $(1.0758)$ & $(1.3570)$ & $(1.2881)$ \\
\hline & \multirow{2}{*}{2.5} & 0.9476 & 0.8730 & 0.8622 & 0.9128 & 0.9348 & 0.9396 \\
\hline & & $(1.6354)$ & $(0.8877)$ & $(0.8907)$ & $(1.0342)$ & $(1.3011)$ & $(1.2200)$ \\
\hline & \multirow[t]{2}{*}{4} & 0.9474 & 0.8532 & 0.8490 & 0.8958 & 0.9294 & 0.9290 \\
\hline & & (1.5569) & $(0.8388)$ & $(0.8411)$ & $(0.9825)$ & (1.2356) & (1.1332) \\
\hline & \multirow{2}{*}{9} & 0.9472 & 0.8552 & 0.8830 & 0.9058 & 0.9402 & 0.9388 \\
\hline & & $(0.6355)$ & $(0.5167)$ & $(0.5229)$ & $(0.5159)$ & $(0.5988)$ & $(0.5770)$ \\
\hline \multirow[t]{12}{*}{$(10,20)$} & \multirow[t]{2}{*}{0.5} & 0.9500 & 0.7112 & 0.8002 & 0.8624 & 0.9398 & 0.9596 \\
\hline & & $(3.6607)$ & $(1.5948)$ & $(1.1618)$ & $(1.7329)$ & $(3.0961)$ & $(2.9601)$ \\
\hline & \multirow[t]{2}{*}{1} & 0.9502 & 0.9366 & 0.9000 & 0.9358 & 0.9394 & 0.9576 \\
\hline & & (1.8261) & (1.0249) & (1.0273) & (1.1529) & $(1.4955)$ & (1.451) \\
\hline & \multirow[t]{2}{*}{2} & 0.9512 & 0.8626 & 0.8586 & 0.9088 & 0.9380 & 0.9370 \\
\hline & & (1.5731) & $(0.8627)$ & $(0.8655)$ & (1.0039) & $(1.2633)$ & (1.1696) \\
\hline & \multirow[t]{2}{*}{2.5} & 0.9470 & 0.8464 & 0.8390 & 0.8936 & 0.9330 & 0.9260 \\
\hline & & (1.5644) & $(0.8484)$ & $(0.8518)$ & $(0.9886)$ & $(1.2476)$ & (1.1432) \\
\hline & \multirow[t]{2}{*}{4} & 0.9604 & 0.8326 & 0.8300 & 0.8782 & 0.9324 & 0.9186 \\
\hline & & (1.5208) & $(0.8193)$ & $(0.8219)$ & $(0.9596)$ & (1.2102) & (1.0956) \\
\hline & \multirow[t]{2}{*}{9} & 0.9544 & 0.8356 & 0.8326 & 0.8840 & 0.9382 & 0.9204 \\
\hline & & (1.5123) & $(0.8095)$ & $(0.8134)$ & $(0.9496)$ & (1.1960) & $(1.0712)$ \\
\hline \multirow[t]{12}{*}{$(20,20)$} & \multirow[t]{2}{*}{0.5} & 0.9538 & 0.6378 & 0.7808 & 0.8274 & 0.9440 & 0.9484 \\
\hline & & (3.2994) & $(1.5021)$ & $(1.5258)$ & (1.5847) & (2.8817) & $(2.6754)$ \\
\hline & \multirow[t]{2}{*}{1} & 0.9478 & 0.9510 & 0.9156 & 0.9420 & 0.9402 & 0.9598 \\
\hline & & $(1.2330)$ & $(\mathbf{0 . 8 7 3 5})$ & $(0.8781)$ & $(0.9134)$ & $(1.1231)$ & (1.1132) \\
\hline & 2 & 0.9514 & 0.9008 & 0.9038 & 0.9346 & 0.9420 & 0.9526 \\
\hline & & $(0.9275)$ & $(0.6855)$ & $(0.6899)$ & $(0.7258)$ & $(0.8490)$ & $(0.8271)$ \\
\hline & 2.5 & 0.9512 & 0.8846 & 0.8936 & 0.9220 & 0.9414 & 0.9460 \\
\hline & & $(0.8922)$ & $(0.6606)$ & $(0.6657)$ & $(0.6971)$ & $(0.8163)$ & $(0.7900)$ \\
\hline & 4 & 0.9506 & 0.8738 & 0.8844 & 0.9186 & 0.9430 & 0.9468 \\
\hline & & $(0.8582)$ & $(0.6286)$ & $(0.6334)$ & $(0.6660)$ & $(0.7847)$ & $(0.7507)$ \\
\hline & 9 & 0.9486 & 0.8564 & 0.8692 & 0.9058 & 0.9370 & 0.9314 \\
\hline & & $(0.8336)$ & $(0.6079)$ & $(0.6133)$ & $(0.6444)$ & $(0.7584)$ & $(0.7186)$ \\
\hline$(30,30)$ & 0.5 & 0.9540 & 0.6384 & 0.8140 & 0.8240 & 0.9494 & 0.9486 \\
\hline & & (2.3309) & $(1.3775)$ & (1.4077) & $(1.2727)$ & (2.1542) & $(2.0485)$ \\
\hline & 1 & 0.9482 & 0.9486 & 0.9278 & 0.9346 & 0.9458 & 0.9600 \\
\hline & & $(0.9231)$ & $(0.7463)$ & $(0.7532)$ & $(0.7347)$ & $(0.8719)$ & $(0.8667)$ \\
\hline & 2 & 0.9570 & 0.9056 & 0.9134 & 0.9316 & 0.9508 & 0.9520 \\
\hline & & (0.7005) & $(0.5752)$ & $(0.5798)$ & $(0.5827)$ & $(0.6629)$ & $(0.6512)$ \\
\hline & 2.5 & 0.9568 & 0.8922 & 0.9074 & 0.9314 & 0.9530 & 0.9536 \\
\hline & & $(0.6806)$ & $(0.5584)$ & $(0.5636)$ & $(0.5635)$ & $(0.6437)$ & $(0.6293)$ \\
\hline & 4 & 0.9484 & 0.8700 & 0.8902 & 0.9090 & 0.9404 & 0.9376 \\
\hline & & $(0.6537)$ & $(0.5371)$ & $(0.5435)$ & $(0.5374)$ & $(0.6176)$ & $(0.5992)$ \\
\hline & 9 & 0.9528 & 0.8602 & 0.8876 & 0.9068 & 0.9436 & 0.9378 \\
\hline & & $(0.6341)$ & $(0.5199)$ & $(0.5262)$ & $(0.5194)$ & $(0.5916)$ & $(0.5747)$ \\
\hline
\end{tabular}


Table 2 (Continued)

\begin{tabular}{|c|c|c|c|c|c|c|c|}
\hline \multirow{2}{*}{$(n, m)$} & \multirow{2}{*}{$k$} & \multicolumn{6}{|c|}{ Coverage probability (Expected length) } \\
\hline & & $C I_{\delta(g c i)}$ & $C I_{\delta(p b)}$ & $C I_{\delta(b s)}$ & $C I_{\delta(m . H R)}$ & $C I_{\delta(M C M C)}$ & $C I_{\delta(H P D)}$ \\
\hline \multirow[t]{12}{*}{$(30,50)$} & \multirow[t]{2}{*}{0.5} & 0.9532 & 0.6688 & 0.8768 & 0.8408 & 0.9496 & 0.9568 \\
\hline & & (1.7003) & (1.2713) & (1.3092) & $(1.0311)$ & $(1.6218)$ & (1.5842) \\
\hline & \multirow[t]{2}{*}{1} & 0.9540 & 0.9416 & 0.9306 & 0.9352 & 0.9500 & 0.9576 \\
\hline & & $(0.8051)$ & $(0.6830)$ & $(0.6899)$ & $(0.6549)$ & $(0.7671)$ & $(0.7609)$ \\
\hline & \multirow[t]{2}{*}{2} & 0.9504 & 0.8884 & 0.9036 & 0.9200 & 0.9464 & 0.9444 \\
\hline & & $(0.6717)$ & $(0.5544)$ & $(0.5595)$ & $(0.5569)$ & $(0.6366)$ & $(0.6212)$ \\
\hline & \multirow[t]{2}{*}{2.5} & 0.9512 & 0.8738 & 0.8988 & 0.9168 & 0.9462 & 0.9412 \\
\hline & & $(0.6576)$ & $(0.5405)$ & $(0.5464)$ & $(0.5436)$ & $(0.6227)$ & $(0.6055)$ \\
\hline & \multirow[t]{2}{*}{4} & 0.9528 & 0.8776 & 0.8914 & 0.9124 & 0.9474 & 0.9436 \\
\hline & & $(0.6435)$ & $(0.5277)$ & $(0.5341)$ & $(0.5288)$ & $(0.6088)$ & $(0.5888)$ \\
\hline & \multirow{2}{*}{9} & 0.9472 & 0.8552 & 0.8830 & 0.9058 & 0.9402 & 0.9388 \\
\hline & & $(0.6355)$ & $(0.5167)$ & $(0.5229)$ & $(0.5159)$ & $(0.5988)$ & $(0.5770)$ \\
\hline \multirow[t]{12}{*}{$(50,50)$} & \multirow[t]{2}{*}{0.5} & 0.9532 & 0.6506 & 0.8538 & 0.8144 & 0.9484 & 0.9532 \\
\hline & & $(1.6366)$ & $(1.2485)$ & $(0.9732)$ & $(0.9766)$ & $(1.5637)$ & (1.5147) \\
\hline & \multirow[t]{2}{*}{1} & 0.9498 & 0.9378 & 0.9380 & 0.9338 & 0.9470 & 0.9564 \\
\hline & & $(0.6698)$ & $(0.6120)$ & $(0.6178)$ & $(0.5627)$ & $(0.6484)$ & $(0.6458)$ \\
\hline & \multirow[t]{2}{*}{2} & 0.9512 & 0.9012 & 0.9158 & 0.9288 & 0.9476 & 0.9486 \\
\hline & & $(0.5152)$ & $(0.4690)$ & $(0.4729)$ & $(0.4476)$ & $(0.4990)$ & $(0.4931)$ \\
\hline & \multirow[t]{2}{*}{2.5} & 0.9498 & 0.8882 & 0.9198 & 0.9300 & 0.9478 & 0.9500 \\
\hline & & $(0.4994)$ & $(0.4542)$ & $(0.4588)$ & $(0.4320)$ & $(0.4834)$ & $(0.4764)$ \\
\hline & \multirow[t]{2}{*}{4} & 0.9484 & 0.8852 & 0.9108 & 0.9212 & 0.9464 & 0.9436 \\
\hline & & $(0.4791)$ & $(0.4367)$ & $(0.4416)$ & $(0.4116)$ & $(0.4637)$ & $(0.4549)$ \\
\hline & \multirow[t]{2}{*}{9} & 0.9496 & 0.8812 & 0.9072 & 0.9142 & 0.9446 & 0.9430 \\
\hline & & $(0.4689)$ & $(0.4254)$ & $(0.4299)$ & $(0.4005)$ & $(0.4520)$ & $(0.4416)$ \\
\hline \multirow[t]{12}{*}{$(50,100)$} & \multirow[t]{2}{*}{0.5} & 0.9546 & 0.7092 & 0.9182 & 0.8404 & 0.9522 & 0.9566 \\
\hline & & $(1.1315)$ & (1.1025) & $(1.1526)$ & $(0.7399)$ & (1.1044) & (1.0913) \\
\hline & \multirow[t]{2}{*}{1} & 0.9506 & 0.9314 & 0.9384 & 0.9272 & 0.9508 & 0.9562 \\
\hline & & $(0.5698)$ & $(0.5402)$ & $(0.5444)$ & $(0.4860)$ & $(0.5548)$ & $(0.5511)$ \\
\hline & 2 & 0.9450 & 0.8936 & 0.9102 & 0.9134 & 0.9414 & 0.9390 \\
\hline & & $(0.4902)$ & $(0.4502)$ & $(0.4552)$ & $(0.4232)$ & $(0.4749)$ & $(0.4670)$ \\
\hline & 2.5 & 0.9530 & 0.8856 & 0.9162 & 0.9254 & 0.9524 & 0.9490 \\
\hline & & $(0.4802)$ & $(0.4380)$ & $(0.4432)$ & $(0.4141)$ & $(0.4659)$ & $(0.4572)$ \\
\hline & 4 & 0.9512 & 0.8882 & 0.9138 & 0.9198 & 0.9470 & 0.9454 \\
\hline & & $(0.4730)$ & $(0.4317)$ & $(0.4369)$ & $(0.4052)$ & $(0.4579)$ & $(0.4482)$ \\
\hline & 9 & 0.9504 & 0.8750 & 0.9034 & 0.9094 & - & - \\
\hline & & $(0.4658)$ & $(0.4256)$ & $(0.4319)$ & $(0.3979)$ & - & - \\
\hline$(100,100)$ & 0.5 & 0.9468 & 0.6818 & 0.9048 & 0.8158 & 0.9496 & 0.9468 \\
\hline & & $(1.0733)$ & $(1.0585)$ & (1.1081) & $(0.6835)$ & $(1.0506)$ & (1.0325) \\
\hline & 1 & 0.9498 & 0.9374 & 0.9524 & 0.9300 & 0.9496 & 0.9512 \\
\hline & & $(0.4518)$ & $(0.4644)$ & $(0.4673)$ & $(0.3944)$ & $(0.4446)$ & $(0.4432)$ \\
\hline & 2 & 0.9552 & 0.9142 & 0.9438 & 0.9364 & 0.9538 & 0.9544 \\
\hline & & $(0.3505)$ & $(0.3527)$ & $(0.3548)$ & $(0.3145)$ & $(0.3449)$ & $(0.3424)$ \\
\hline & 2.5 & 0.9530 & 0.9043 & 0.9424 & 0.9294 & 0.9504 & 0.9504 \\
\hline & & $(0.3393)$ & $(0.3447)$ & $(0.3475)$ & $(0.3032)$ & $(0.3340)$ & $(0.3310)$ \\
\hline & 4 & 0.9472 & 0.8916 & 0.9320 & 0.9166 & 0.9446 & 0.9428 \\
\hline & & $(0.3259)$ & $(0.3323)$ & $(0.3351)$ & $(0.2889)$ & $(0.3206)$ & $(0.3170)$ \\
\hline & 9 & 0.9484 & 0.8944 & 0.9304 & 0.9170 & - & - \\
\hline & & $(0.3189)$ & $(0.3253)$ & $(0.3286)$ & $(0.2810)$ & - & - \\
\hline
\end{tabular}




\section{EMPIRICAL APPLICATION OF THE DERIVED CONFIDENCE INTERVALS}

\section{Example 1}

Wind speed data set were collected at 90-meter wind energy potential stations in Trad and Chonburi provinces in 2016 by the Department of Alternative Energy Development and Efficiency, Ministry of Energy (https://www. dede.go.th/ewt_news.php?nid=47706). These are coastal provinces in eastern Thailand that have been identified as potential areas for harvesting wind energy. Figure 3 shows Q-Q plots of the Weibull distributions of the data from the two provinces. Furthermore, the Akaike Information Criterion (AIC) and Bayesian Information Criterion (BIC) values reported in Table 3 indicate that these two datasets fit Weibull distributions because the latter have the smallest values for both criteria.

The statistical summary of the estimates of the parameters for these datasets is $(n, m)=(12,11), \hat{a}_{X}=$ $1.7621, \hat{a}_{Y}=4.5839, \hat{k}_{X}=1.4832, \hat{k}_{Y}=9.0794, \hat{\lambda}_{X}=0.6860$ and $\hat{\lambda}_{Y}=0.1317$, while the actual difference between the coefficients of variation of the two Weibull distributions $\hat{\delta}=0.5543$. The confidence intervals based on the proposed methods are given in Table 4. The results show that the Bayesian-HPD interval performed well in terms of the coverage probability and expected length when the sample sizes are small, which supports the simulation results. Finally, Figure 4 shows a trace plot of the generated $\delta$ value.

A

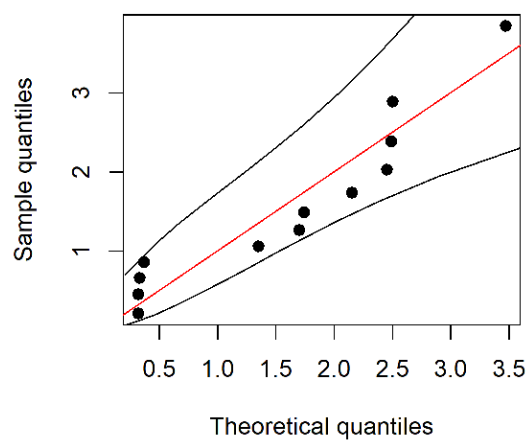

B

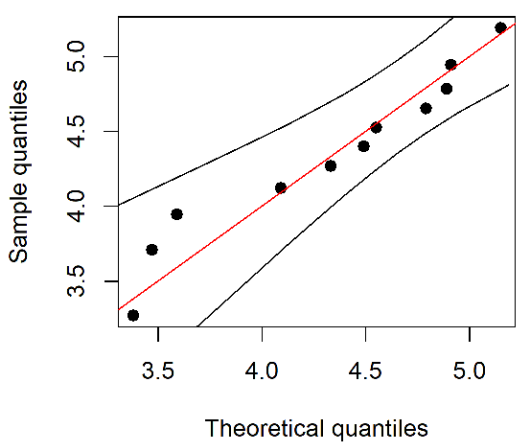

Figure 3. The Weibull Q-Q plot for the wind speed data of two provinces, Thailand: (A) Trad Province (B) Chonburi Province.

Table 3. The AIC and BIC values of the wind speed data of Trad province and Chonburi province.

\begin{tabular}{lcccc}
\hline Method & \multicolumn{2}{c}{ Trad } & \multicolumn{2}{c}{ Chonburi } \\
\cline { 2 - 5 } & AIC & BIC & AIC & BIC \\
\hline Weibull & $\mathbf{3 7 . 0 2 7 2}$ & $\mathbf{3 7 . 9 9 7 0}$ & $\mathbf{2 2 . 8 0 5 4}$ & $\mathbf{2 3 . 6 0 1 1}$ \\
Exponential & 37.2675 & 37.7524 & 56.2471 & 56.6450 \\
Gamma & 37.5735 & 38.5433 & 24.0780 & 24.8737 \\
Log-normal & 39.1594 & 40.1292 & 24.3327 & 25.1285 \\
\hline
\end{tabular}

\section{Example 2}

Data on wind speeds measured at 90-meter wind energy potential stations in the southern and northeastern regions of Thailand were collected in April-May 2019 by the Department of Alternative Energy Development and Efficiency, Ministry of Energy (https://www.dede.go.th/more_news.php?cid= 501). The summary statistics for the southern region are $n=12, \hat{a}_{X}=2.5334, \hat{k}_{X}=2.3956$ and $\hat{\lambda}_{X}=$ 0.4441 and the northeastern region are $m=20, \hat{a}_{Y}=4.4608, \hat{k}_{Y}=3.8457$ and $\hat{\lambda}_{Y}=0.2906$. The actual difference between the coefficients of variation of the Weibull distributions of these datasets $\hat{\delta}=0.1538$. Weibull Q-Q plots of these data are shown in Figure 5 and the correctness of fitting the data to Weibull distributions in terms of the smallest AIC and BIC values are reported in Table 5. The 95\% confidence intervals for $\delta$ by applying the six methods (Table 6) indicate that the Bayesian-HPD interval performed 
Table 4. The 95\% confidence intervals for the difference between the coefficients of variation of the wind speed data of Trad and Chonburi provinces, Thailand.

\begin{tabular}{cccc}
\hline \multirow{2}{*}{ Method } & \multicolumn{3}{c}{ Confidence intervals for $\delta$} \\
\cline { 2 - 4 } & Lower & Upper & Length \\
\hline GCI & 0.3316 & 1.1013 & 0.7697 \\
MOVER & 0.2398 & 0.8689 & 0.6291 \\
PB & 0.2081 & 0.8036 & 0.5155 \\
BS & 0.2531 & 0.8555 & 0.6024 \\
MCMC & 0.2912 & 1.0095 & 0.7183 \\
HPD & 0.2600 & 0.9216 & 0.6616 \\
\hline
\end{tabular}

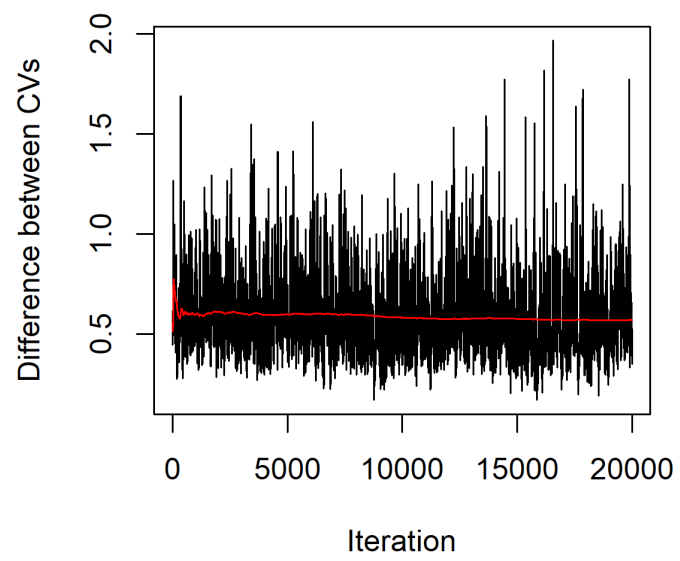

Figure 4. Plot of generated $\delta$ of example 1 vs. iteration of the MCMC algorithm.

well in terms of the coverage probability and expected length when the difference between the coefficients of variation was small, which is once again consistent with the simulation study results. Finally, the trace plot of the generated $\delta$ value are presented in Figure 6 .

A

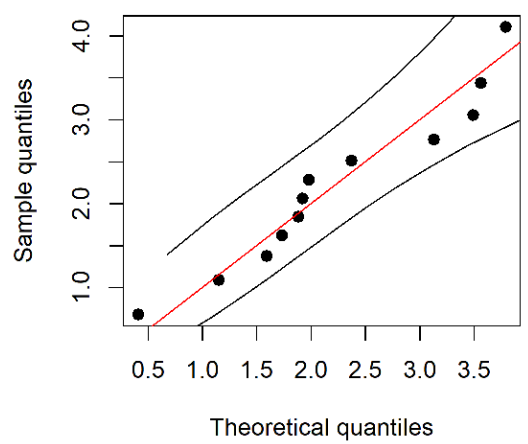

B

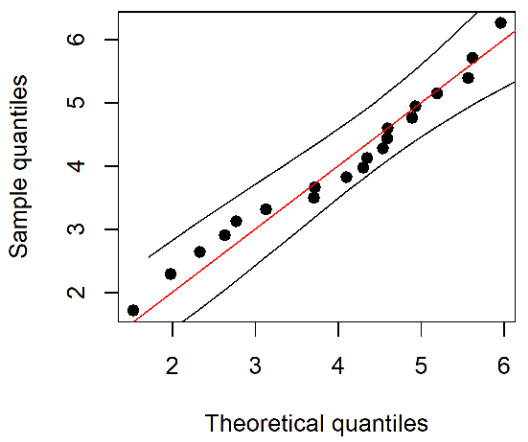

Figure 5. The Weibull Q-Q plot for the wind speed data of two Thailand's regions: (A) Southern (B) Northeastern. 
Table 5. The AIC and BIC values of the wind speed data of the southern and northeastern regions of Thailand.

\begin{tabular}{lcccc}
\hline Method & \multicolumn{2}{c}{ Southern } & \multicolumn{2}{c}{ Northeastern } \\
\cline { 2 - 5 } & AIC & BIC & AIC & BIC \\
\hline Weibull & $\mathbf{3 7 . 9 1 7 6}$ & $\mathbf{3 8 . 8 8 7 1}$ & $\mathbf{6 8 . 3 7 5 8}$ & $\mathbf{7 0 . 3 6 7 3}$ \\
Exponential & 45.4623 & 45.9472 & 97.6711 & 98.6669 \\
Gamma & 39.2535 & 75.5790 & 71.5482 & 73.5397 \\
Log-normal & 41.5439 & 42.5137 & 73.5875 & 40.2233 \\
\hline
\end{tabular}

Table 6. The 95\% confidence intervals for the difference between the coefficients of variation of the wind speed data of the southern and northeastern regions of Thailand.

\begin{tabular}{cccc}
\hline Method & \multicolumn{3}{c}{ Confidence intervals for $\delta$} \\
\cline { 2 - 4 } & Lower & Upper & Length \\
\hline GCI & -0.0270 & 0.4807 & 0.5077 \\
MOVER & -0.0678 & 0.3755 & 0.4435 \\
PB & -0.0450 & 0.3763 & 0.4213 \\
BS & -0.0553 & 0.3630 & 0.4183 \\
MCMC & -0.0241 & 0.4727 & 0.4768 \\
HPD & -0.0445 & 0.4267 & 0.4712 \\
\hline
\end{tabular}

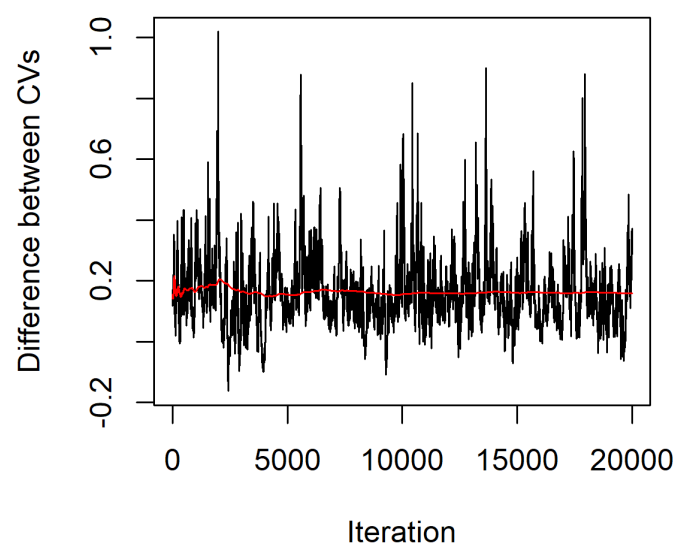

Figure 6. Plot of generated $\delta$ of example 2 vs. iteration of the MCMC algorithm.

\section{DISCUSSION}

It can be seen from the results of the study that GCI performed well in almost all cases since its coverage probability was greater than or close to the nominal confidence level. For the Bayesian methods, BayesianMCMC and Bayesian-HPD Interval produced similar results and performed well when $k_{Y}$ (the difference between the coefficients of variation) was small, zero, or negative. For large sample sizes, the Bayesian methods were not suitable when $k_{Y}$ was large. Moreover, the expected length of the Bayesian-HPD interval was always shorter than Bayesian-MCMC. In relation to the expected lengths, they tended to decrease when the sample sizes and/or $k_{Y}$ increased. 


\section{CONCLUSIONS}

This paper proposed six methods for deriving the confidence interval for the difference between the coefficients of variation of Weibull distributions: the GCI method, the Bayesian-MCMC method, the Bayesian-HPD interval, MOVER based on Hendricks and Robey's confidence interval, the percentile bootstrap method, and the bootstrap confidence interval with standard errors. The performances of these methods were compared using the coverage probability and the expected length. The results of simulation studies showed that the GCI method and the Bayesian-HPD method were the best in different scenarios. The Bayesian-HPD was preferable when shape parameter $k_{y}$ was small. The percentile bootstrap method can be used when the sample sizes are small and $k_{y}=1$. However, MOVER based on Hendricks and Robey's confidence interval and the bootstrap confidence interval with standard errors are not recommended since these methods yielded coverage probabilities under the nominal confidence level of 0.95 for almost all cases.

\section{REFERENCES}

Banik, S. and Kibria, B. G. (2011). Estimating the population coefficient of variation by confidence intervals. Communications in Statistics-Simulation and Computation, 40(8):1236-1261.

Box, G. E. and Tiao, G. C. (2011). Bayesian inference in statistical analysis., volume 40. John Wiley \& Sons.

Buntao, N. and Niwitpong, S.-a. (2013). Confidence intervals for the difference of coefficients of variation for lognormal distributions and delta-lognormal distributions. Applied Mathematical Sciences, 6:6691-6704.

Chaichana, T., Chaiyatep, S., Jompakdee, W., Klongpanit, W., and Dussadee, N. (2015). Analysis of wind power potential in the rainy season at the high agricultural project under the royal initiative at mon lan using weibull distribution. In TISD 2008 the 2nd technology and innovation for sustainable development conference. Klungnana Vittaya Press.

Chancham, C., Klompong, N., Puangkaew, W., and Waewsak, J. (2009). An analysis of wind potential at huasai in nakhon si thammarat province. ASEAN Journal of Scientific and Technological Reports, 12(2):10-19.

Cohen, A. C. (1965). Maximum likelihood estimation in the weibull distribution based on complete and on censored samples. Technometrics, 7:579-588.

Colosimo, E. A. and Ho, L. L. (1999). Practical approach to interval estimation for the weibull mean lifetime. Quality Engineering., 12(2):161-167.

Donner, A. and Zou, G. Y. (2010). Closed-form confidence intervals for functions of the normal mean and standard deviation. Stat Methods Med Res, 21:347-359.

Efron, B. and Tibshirani, R. J. (1993). An introduction to the bootstrap chapman \& hall. New York, 436.

Faber, D. S. and Korn, H. (1991). Applicability of the coefficient of variation method for analyzing synaptic plasticity. Biophysical journal, 60(5):1288-1294.

Geman, S. and Geman, D. (1984). Stochastic relaxation, gibbs distributions, and the bayesian restoration of images. IEEE Transactions on pattern analysis and machine intelligence, pages 721-741.

Green, E. J., Roesch Jr, F. A., Smith, A. F. M., and Strawderman, W. E. (1994). Bayesian estimation for the three-parameter weibull distribution with tree diameter data. Biometric Society, pages 254-269.

Hendricks, W. A. and Robey, W. K. (1936). The sampling distribution of the coefficient of variation. Annals of Mathematical Statistics, 17:129-132.

Ibrahim, N. A. (2010). Bayesian estimator for weibull distribution with censored data using extension of jeffrey prior information. Procedia-Social and Behavioral Sciences, 8:663-669.

Krishnamoorthy, K. and Lin, Y. (2010). Confidence limits for stress-strength reliability involving weibull models. Journal of Statistical Planning and Inference, 140:1754-1764.

Krishnamoorthy, K., Lin, Y., and Xia, Y. (2009). Confidence limits and prediction limits for a weibull distribution based on the generalized variable approach. Journal of Statistical Planning and Inference, 139:2675-2684.

Krishnamoorthy, K., Mukherjee, S., and Guo, H. (2007). Inference on reliability in two-parameter exponential stress-strength models. Metrika, 65:261-273.

Kundu, D. and Howlader, H. (2010). Bayesian inference and prediction of the inverse weibull distribution for type-ii censored data. Computational Statistics \& Data Analysis, 54(6):1547-1558. 
La-ongkaew, M., Niwitpong, S.-A., and Niwitpong, S. (2019). Confidence Intervals for Single Coefficient of Variation of Weibull Distribution. In Proceedings of the 3rd International Conference on Vision, Image and Signal Processing, pages 1-6.

Mahmoudvand, R. and Hassani, H. (2009). Two new confidence intervals for the coefficient of variation in a normal distribution. Journal of Applied Statistics, 36:429-442.

Menon, M. (1963). Estimation of the shape and scale parameters of the weibull distribution. Technometrics, 5(2):175-182.

Niwitpong, S.-a. (2013). Confidence interval for coefficient of variation of lognormal distribution with restricted parameter space. Applied Mathematical Sciences, 7(77):3805-3810.

Pang, W. K., Forster, J. J., and Troutt, M. D. (2001). Estimation of wind speed distribution using markov chain monte carlo techniques. Journal of Applied Meteorology, 40(8):1476-1484.

Pang, W. K., Leung, P. K., Huang, W. K., and Liu, W. (2005). On interval estimation of the coefficient of variation for the three-parameter weibull, lognormal and gamma distribution:a simulation-based approach. European Journal of Operational Research, 164(2):367-377.

Sangnawakij, P., Niwitpong, S.-a., and Niwitpong, S. (2015). Confidence intervals for the ratio of coefficients of variation of the gamma distributions. In Integrated Uncertainty in Knowledge Modelling and Decision Making, Lecture Notes in Computer Science. Cham: Springer, volume 9376, pages 193-203. Springer, Cham.

Saraiva, E. F. and Suzuki, A. K. (2017). Bayesian computational methods for estimation of two-parameters weibull distribution in presence of right-censored data. Chilean Journal of Statistics (ChJS), 8(2).

Thoman, D. R., Bain, L. J., and Antle, C. E. (1969). Inferences on the parameters of the weibull distribution. Technometrics, 11:445-446.

Tian, L. (2005). Inferences on the common coefficient of variation. Statistics in Medicine, 24:2213-2220.

Vangel, M. (1996). Confidence intervals for a normal coefficient of variation. The American Statistician, $50(1): 21-26$.

Weerahandi, S. (1993). Generalized confidence intervals. Journal of American Statistical, 88:899-905.

Weibull, W. (1951). A statistical distribution function of wide applicability. Journal of applied mechanics, 18(3):293-297.

Yalcinkaya, M. and Birgoren, B. (2017). Confidence interval estimation of weibull lower percentiles in small samples via bayesian inference. Journal of the European Ceramic Society, 37:2983-2990.

Yosboonruang, N., Niwitpong, S.-a., and Niwitpong, S. (2019). Measuring the dispersion of rainfall using bayesian confidence intervals for coefficient of variation of delta-lognormal distribution: a study from thailand. PeerJ.

Yosboonruang, N., Niwitpong, S.-a., and Niwitpong, S. (2020). The bayesian confidence intervals for measuring the difference between dispersions of rainfall in thailand. PeerJ. 